\title{
Aridification: l'effet du changement climatique dans le Bassin Carpathique
}

Aridification: The effect of Climate Change in the Carpathian Basin

Aridifizierung : Auswirkung des Klimawandels im Karpatenbecken

Ádám Kertész, Dénes Lóczy et Tamás Huszár

\section{(2)enEdition}

Journals

Édition électronique

URL : http://journals.openedition.org/rge/3880

DOI : $10.4000 /$ rge.3880

ISSN : 2108-6478

Éditeur

Association des géographes de l'Est

Édition imprimée

Date de publication : 1 septembre 2001

ISSN : 0035-3213

Référence électronique

Ádám Kertész, Dénes Lóczy et Tamás Huszár, « Aridification: l'effet du changement climatique dans le Bassin Carpathique », Revue Géographique de l'Est [En ligne], vol. 41 / 4 | 2001, mis en ligne le 22 juillet 2013, consulté le 08 septembre 2020. URL : http://journals.openedition.org/rge/3880 ; DOI : https:// doi.org/10.4000/rge.3880

Ce document a été généré automatiquement le 8 septembre 2020

Tous droits réservés 


\title{
Aridification: l'effet du changement climatique dans le Bassin Carpathique
}

\author{
Aridification: The effect of Climate Change in the Carpathian Basin \\ Aridifizierung : Auswirkung des Klimawandels im Karpatenbecken
}

Ádám Kertész, Dénes Lóczy et Tamás Huszár

\section{Introduction}

1 Si la notion de changement climatique global est aujourd'hui admise, le détail de ses processus et de ses conséquences reste à établir. Une de ses manifestations les plus nettes est l'assèchement des régions méditerranéennes, qui peut même prendre les proportions d'une désertification en certains endroits où la dégradation des terres cultivables a atteint un degré critique.

La Hongrie, pays de climat « tempéré » aux influences atlantiques et continentales, présente également des caractéristiques méditerranéennes au Sud de la Transdanubie, caractéristiques qui se sont renforcées depuis un siècle (Molnár, 1995). Il est probable que la légère tendance au réchauffement et la tendance relativement forte à l'assèchement que l'on observe dans les données climatiques de la Hongrie soient également à mettre en rapport avec le changement climatique mondial.

Cette importante question a fait l'objet d'études dans le cadre du programme MEDALUS (Mediterranean Desertification and Land Use) de l'Union Européenne, dont le but était de modéliser la transformation probable du milieu naturel et social et d'élaborer en réponse les stratégies d'un développement durable. Les recherches ont été étendues à la Hongrie au cours des projets MEDALUS II (1994-1996) et MEDALUS III (1996-1998), sous le nom de "Programme de recherches sur l'aridification de la Hongrie », sousprojet MEDEAST, dont les objectifs étaient les suivants (fig. 1 et 2 ) : 
Figure 1 : Schéma général du programme MEDALUS

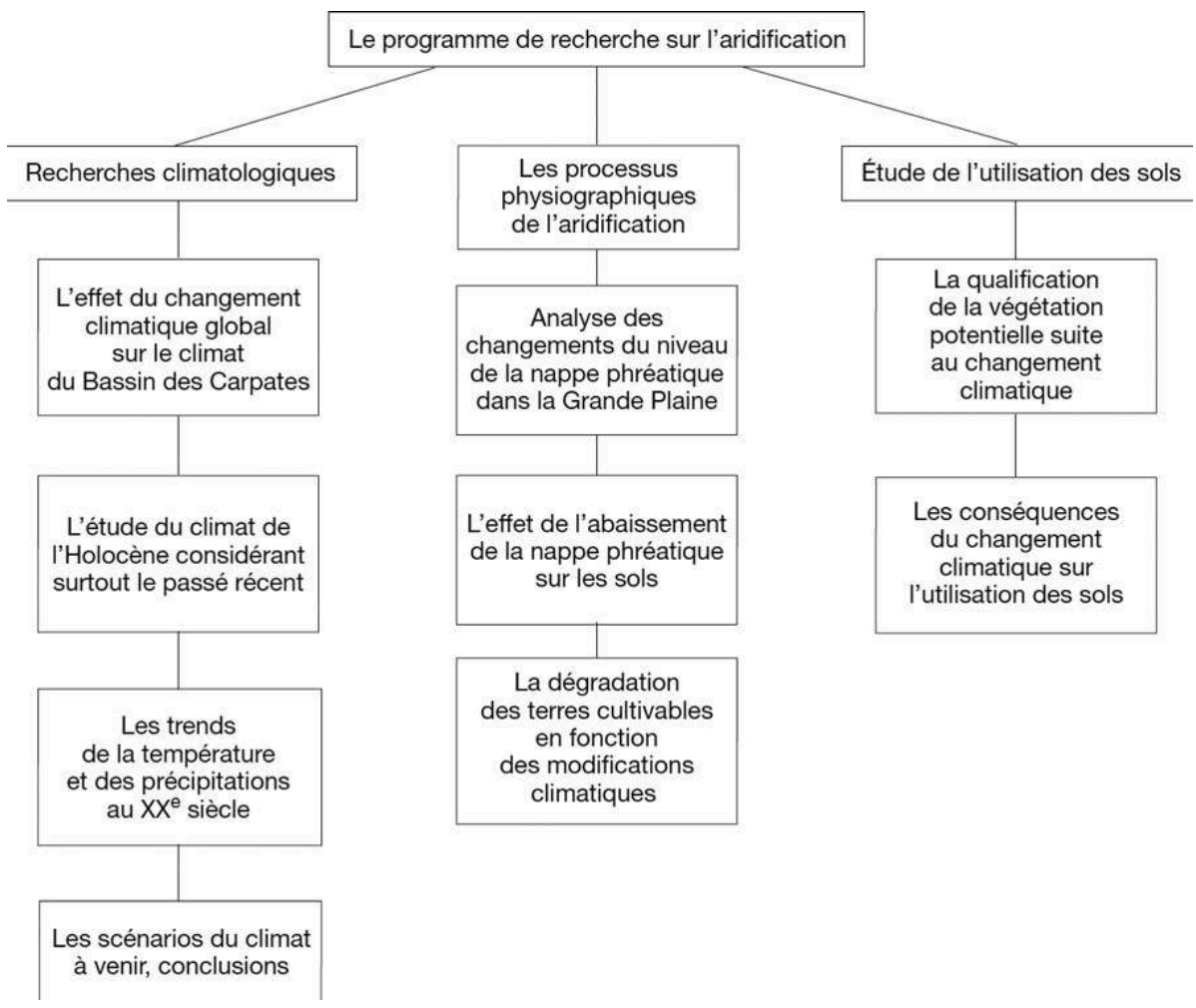

Figure 2 : Régionalisation du programme dans le Bassin carpathique

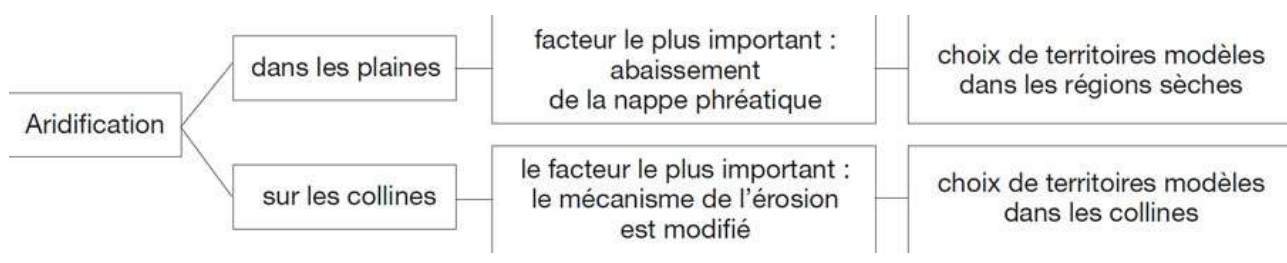

4 1. Effectuer des recherches climatologiques pour découvrir les effets du changement climatique global sur le territoire hongrois; en examinant la durée de la variation climatique (courte ou moyenne), essayer de savoir s'il s'agit bien d'une tendance climatique à l'assèchement et non d'une simple oscillation; ensuite, en tenant compte des effets de l'impact anthropique, essayer d'élaborer le scénario du changement climatique à venir ;

5 2. Effectuer des recherches sur les conséquences physiographiques de l'assèchement: celles-ci se manifestent essentiellement sur les nappes phréatiques souterraines, sur les sols cultivables et sur la végétation.

6 Pour ce qui concerne les nappes, il fallait observer et enregistrer les variations de niveau des eaux souterraines dans la Grande Plaine, plus particulièrement entre le Danube et la Tisza, régions les plus frappées par la sécheresse, et savoir depuis quand le changement s'était produit.

7 En ce qui concerne les sols et la végétation, il s'agissait d'étudier l'évolution de leur teneur en humidité en rapport avec celle du niveau des eaux souterraines, mais aussi d'interpréter les différents résultats relatifs aux mesures d'érosion depuis un passé proche. Ainsi, des recherches ont été entreprises pour analyser comment les 
précipitations extrêmes (qui augmentent avec l'aridité) influencent le ruissellement superficiel ainsi que le dépôt des sédiments sur les pentes et en fonds de vallées. De même, les changements intervenus dans l'utilisation des sols peuvent être considérés comme des conséquences de l'aridification et peuvent être cartographiés, parallèlement aux modifications de la couverture végétale naturelle.

3. Le troisième volet des recherches concerne l'utilisation du sol; partant de l'utilisation actuelle, il convient de proposer les éventuels réajustements nécessaires. Cette phase conduit à une indispensable régionalisation du programme, distinguant les plaines des régions de collines ou de montagnes; sur ces dernières, aux dénivellations notables, c'est la question de l'érosion et de la dégradation des sols cultivables qui domine; dans les plaines en revanche, le problème principal est le manque d'eau, donc l'assèchement. Des territoires d'étude différents ont donc été retenus, dans le haut pays du Lac Balaton (bassin versant de Örvényesi-Séd et parcelles d'expérimentation de la station de Csákvár) pour la montagne d'une part, interfluve Danube-Tisza pour la plaine d'autre part.

\section{Le changement climatique}

\section{A. Le changement global depuis 1881}

9 Pour mettre en évidence un éventuel changement climatique, il faut étudier l'évolution des deux paramètres fondamentaux, températures et précipitations (Molnár, Mika, 1997). Les changements observés pour ces deux facteurs peuvent permettre dans une certaine mesure de déduire les modifications probables des autres éléments du climat. La Hongrie a la chance de posséder des observations sur ces deux données depuis 1881. On a donc utilisé comme base de données initiale les observations enregistrées sur la période 1881-1989 dans 16 stations météorologiques pour les températures et dans 17 stations pour les précipitations. Sur ces 17 stations, 8 se trouvent dans la Grande Plaine, 2 dans la Petite Plaine, 3 dans l'Ouest de la Hongrie, 2 dans les collines de la Transdanubie, 1 dans les Massifs du Nord, 1 à Budapest : l'ensemble des grandes régions naturelles de la Hongrie est donc bien représenté.

Les diagrammes de températures moyennes annuelles et ceux des précipitations moyennes annuelles montrent deux faits majeurs :

- la variabilité inter-annuelle est très forte : les écarts de 20 à 30 \% par rapport à la moyenne générale sont fréquents ;

- c'est la variabilité des précipitations qui est toujours supérieure à celle des températures.

11 Dans le détail, pour chaque station, on a effectué une étude du sens des variations des températures et des précipitations par une analyse de régression, puis on a déterminé statistiquement la valeur de la tendance moyenne de variation (trend) de la température (Tableau 1) et celle des précipitations (Tableau 2). En dessous d'une valeur statistique critique $\mathrm{T}=1,983$, le niveau de fiabilité à $95 \%$ des résultats n'est pas atteint. 
Tableau 1 : Trends de la température de 1881 à 1989 dans les stations hongroises (séries de données corrigées)

\begin{tabular}{|l|c|c|c|}
\hline Stations & $\begin{array}{c}\text { Trends de la température } \\
\left({ }^{\circ} \mathrm{C} / \mathrm{an}\right)\end{array}$ & $\begin{array}{c}\text { Valeur } \\
\text { critique T }\end{array}$ & Niveau de fiabilité à $95 \%$ \\
\hline Nyíregyháza & 0,011 & 5,532 & oui \\
Zalaegerszeg & 0,011 & 5,307 & oui \\
Debrecen & 0,011 & 5,707 & oui \\
Baja & 0,011 & 5,718 & oui \\
Kalocsa & 0,011 & 5,727 & oui \\
Pápa & 0,011 & 5,330 & oui \\
Pécs & 0,011 & 5,545 & oui \\
Budapest & 0,011 & 5,806 & oui \\
Kecskemét & 0,011 & 5,332 & oui \\
Keszthely & 0,010 & 5,490 & oui \\
Mosonmagyaróvár & 0,010 & 5,307 & oui \\
Szombathely & 0,010 & 5,372 & oui \\
Sopron & 0,010 & 5,174 & oui \\
Szarvas & 0,010 & 4,635 & oui \\
Túrkeve & 0,010 & 4,795 & oui \\
Szeged & 0,010 & 4,519 & oui \\
\multicolumn{1}{|c|}{ Moyenne } & 0,0105 & & \\
\hline
\end{tabular}

Tableau 2 : Trends des précipitations de 1881 à 1989 dans les stations hongroises

\begin{tabular}{|l|c|c|c|}
\hline Stations & $\begin{array}{c}\text { Trends des précipitations } \\
(\mathrm{mm} / \mathrm{an})\end{array}$ & $\begin{array}{c}\text { Valeur } \\
\text { critique T }\end{array}$ & Niveau de fiabilité à $95 \%$ \\
\hline Pécs & $-2,30$ & 5,765 & oui \\
Szombathely & $-1,41$ & 4,432 & oui \\
Sopron & $-1,22$ & 3,518 & oui \\
Budapest & $-1,09$ & 3,309 & oui \\
Nyíregyáza & $-1,02$ & 3,086 & oui \\
Túrkeve & $-0,98$ & oui \\
Szeged & $-0,91$ & 2,980 & oui \\
Kecskemét & $-0,86$ & 3,002 & oui \\
Szarvas & $-0,79$ & 2,892 & oui \\
Baja & $-0,71$ & 2,754 & oui \\
Mosonmagyaróvár & $-0,61$ & 2,070 & non \\
Zalaegerszeg & $-0,56$ & 1,953 & non \\
Debrecen & $-0,47$ & 1,526 & non \\
Kalocsa & $-0,43$ & 1,358 & non \\
Keszthely & $-0,39$ & 1,436 & non \\
Pápa & $-0,27$ & 0,994 & non \\
Eger & $-0,47$ & 0,745 & non \\
\multicolumn{1}{|c|}{ Moyenne } & $-0,918$ & 1,618 & \\
\hline
\end{tabular}

Les calculs montrent ainsi une tendance moyenne générale à l'élévation des valeurs des températures de 0,0104 à $0,0105^{\circ} \mathrm{C} / \mathrm{an}$. Cette élévation des températures moyennes est donc prouvée statistiquement; cependant, d'une part la période observée de 110 ans est encore trop courte pour pouvoir en tirer des conséquences certaines sur le sens de la variation et d'autre part cela ne nous renseigne pas sur les facteurs de l'élévation des températures, qui sont complexes. En tout état de cause, ces «trends» de la 
température montrés par les stations hongroises correspondent aux trends enregistrés en plusieurs autres endroits du globe.

Dans chacune des 17 stations examinées, la diminution des précipitations a été constatée (Tableau 2). Ce trend décroissant peut être accepté à un niveau de confiance de $95 \%$ pour 10 stations. Le trend moyen calculé est de 0,917 à 0,918 mm/an. Cela signifie qu'au cours des 110 dernières années, la diminution des précipitations en Hongrie a été de l'ordre de $100 \mathrm{~mm}$, ce qui représente au moins $15 \%$ de la moyenne nationale des précipitations annuelles : il s'agit donc bien d'un changement considérable.

Ces constatations sont en bon accord avec celles de la bibliographie hongroise et internationale à propos du changement climatique. Elles vont dans le sens de l'idée générale attribuant à des processus globaux l'élévation progressive de la température dans la zone tempérée et à l'affaiblissement (ou à la modification ?) des circulations zonales la diminution des précipitations.

\section{B. Deux tendances particulières, au niveau des saisons et des deux dernières décennies}

\section{Des hivers plus doux et des étés plus frais et plus secs}

Les tendances générales de la période 1881-1989 sont également illustrées par de nombreuses observations courantes; depuis la première moitié du XXe siècle, de nombreux signes du réchauffement se manifestent en Hongrie: ce sont les températures du semestre hivernal qui s'élèvent surtout (mais si les hivers s'adoucissent, les étés deviennent plus frais ; ainsi, la période végétative s'allonge). Les précipitations baissent, ce qui fait que l'humidité du sol et les réserves d'eau accumulées au début de la période végétative diminuent, provoquant une aggravation de la sécheresse estivale.

\section{Une accélération du phénomène depuis 1980 ?}

Deux exemples permettent de constater que la tendance vers l'assèchement s'est amplifiée depuis les deux dernières décennies.

En plusieurs endroits situés entre Danube et Tisza, 1980 est la dernière année où les précipitations annuelles ont atteint la moyenne des 30 années précédentes. Les mêmes vingt dernières années ont vu la température moyenne de la période végétative augmenter de 3 à $5 \%$. Toujours depuis 1980, la forte diminution des précipitations en hiver n'a permis l'infiltration qu'à deux reprises; encore s'agissait-il d'infiltrations insignifiantes, n'autorisant pas la reconstitution de la nappe phréatique: c'est seulement lors de l'hiver 1995-96 que le sol s'est suffisamment imbibé d'eau. Nous verrons plus loin les conséquences de cette situation sur le niveau des nappes.

18 C'est une des raisons qui font que la base de données utilisée pour l'examen des variations des capacités productives du sol, établie sur la période de référence 1940-1970 (Infra), n'est pas représentative du véritable assèchement actuel. 


\section{Les conséquences du changement climatique}

\section{A. L'abaissement du niveau des nappes phréatiques}

19 Comme le montre la figure 3, le niveau des nappes phréatiques profondes a baissé de près de 3 mètres entre 1958 et 1992 sur les terrasses sableuses situés entre Danube et Tisza. Les ressources en eaux profondes ont donc diminué notablement. Le phénomène affecte également les nappes superficielles ; tant que l'oscillation ne dépasse pas $0,50 \mathrm{~m}$ pour ces dernières, les besoins en eau de la majorité des plantes sont satisfaits. Mais un abaissement plus marqué du niveau de la nappe superficielle menace la rentabilité des cultures (Varga-Hasznosits, Harnos, 1988).

Figure 3 : Valeurs moyennes mensuelles du niveau de la nappe phréatique souterraine entre 1952-1992 dans le puits d'observation n816 (Ágasegyháza)

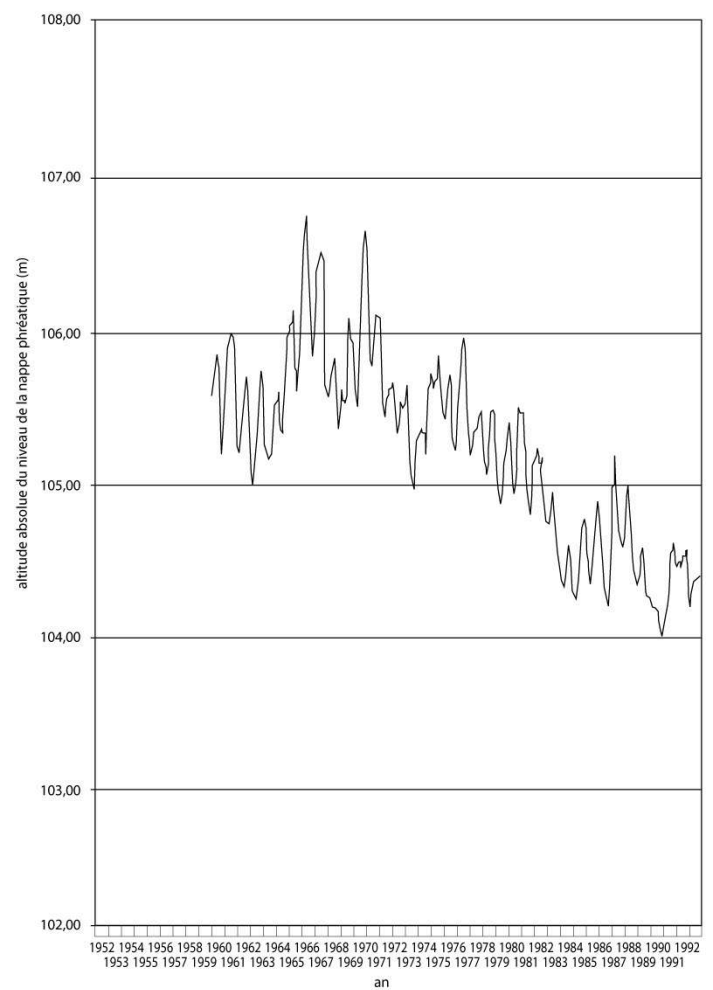

La cause principale de l'abaissement du niveau des eaux souterraines est le retour périodique de la sécheresse, mais l'influence anthropique n'est pas négligeable, car l'approvisionnement des agglomérations exige de plus en plus d'eau, tout comme l'irrigation. Dans la Grande Plaine, on a calculé les parts respectives de ces différents facteurs dans l'abaissement des nappes souterraines : la décroissance des précipitations et l'augmentation de l'évaporation en sont responsables à raison de $50 \%$; la consommation d'eau à raison de $25 \%$, la destruction des forêts et les modifications de l'utilisation du sol interviennent pour $10 \%$ (nous verrons toutefois plus loin que le rôle de la forêt reste très difficile à évaluer); le reste de la baisse est dû à la fois aux modifications des conditions d'écoulement (7\%), à l'utilisation directe des eaux souterraines sans restitution ( $2 \%$ ) et à la réduction de l'alimentation de la nappe par les eaux du Danube et de la Tisza (6\%). 
21 C'est sur l'interfluve Danube-Tisza, où la baisse des précipitations affecte une large surface, que l'abaissement du niveau des nappes phréatiques atteint les proportions les plus fortes et que les conséquences sont les plus dramatiques. Au Sud de Kecskemét, le cumul des déficits hydriques moyens des 15 dernières années dépasse $1 \mathrm{~m}$. Les terrasses sableuses de ce secteur constituent les régions les plus continentales et les plus sèches de la Hongrie, où l'approvisionnement en eau est donc un facteur critique vis-à-vis du potentiel agro-écologique. Il faut ajouter le facteur anthropique depuis plus d'un siècle, d'abord avec la régulation des débits des deux cours d'eau et les drainages dans la seconde moitié du XIXe siècle, puis avec la multiplication des pompages depuis les années 50.

\section{B. Les changements pédologiques}

\section{L'assèchement des sols}

Le changement climatique se traduit directement dans les sols par une diminution de leur teneur en humidité (Várallyay, 1994). Mais cette diminution varie en fonction de la nature du sol: dans les sols très humifères et à grande capacité de tamponnement, elle est beaucoup moins rapide que dans les sols sableux de l'interfluve Danube-Tisza. Ces derniers sont représentés surtout sur des collines dunaires un peu plus élevées que les zones basses hydromorphes, où les sols finement stratifiés sont caractérisés par des processus de salinisation. Autour des lacs saumâtres, alimentés par les eaux souterraines et asséchés partiellement ou définitivement, on peut observer que les échanges entre eaux souterraines et couches humiques du sol sont interrompus; on évolue vers des sols de type solontschak (Kertész et al., 1998). Sur ces sols, un lessivage peut se produire à quelques occasions, quand les rares averses transportent les sels sodiques jusqu'à la nappe phréatique abaissée. La capacité de résistance à la sécheresse de ce type de sol des zones basses est finalement plus élevée que celle des sols sableux et humiques du sommet des collines dunaires. Il n'empêche que la transformation rapide des sols affecte à peu près la moitié de la région.

\section{2. Évolution de la capacité productive des sols}

La notion de capacité productive (land capability) d'un sol est représentée par le nombre d'espèces de plantes cultivables sur un territoire au niveau habituel de la technique agricole, tenant compte de l'ordre d'utilité de ces plantes (McRae, Burnham, 1981).

Les études agro-écologiques de l'Institut de Recherches Géographiques de l'Académie des Sciences ont pris en compte 23 paramètres (Tableau 3) pour évaluer la capacité productive des sols. La qualification des données écologiques a été effectuée à l'aide du système informatique ARC/INFO pour évaluer les besoins des plantes cultivées sur les plus grands emblavements (froment, maïs, tournesol, luzerne, betterave), mais en utisant les données de 1940 à 1970, ce qui fait que cette base de données est établie sur des conditions antérieures au processus d'assèchement actuel. Chaque donnée a fait l'objet d'une traduction cartographique, puis l'ensemble des cartes par paramètre superposées dans le système vectoriel ARC/INFO a fourni des cartes de capacité productive du sol relative à chaque plante. Ces dernières, unifiées, ont abouti à une 
carte générale sur laquelle on peut distinguer des régions ayant à peu près les mêmes caractéristiques écologiques, ce qui permet de définir des zones agro-écologiques.

Tableau 3 : Paramètres utilisés pour évaluer la capacité productive des sols dans les plaines hongroises

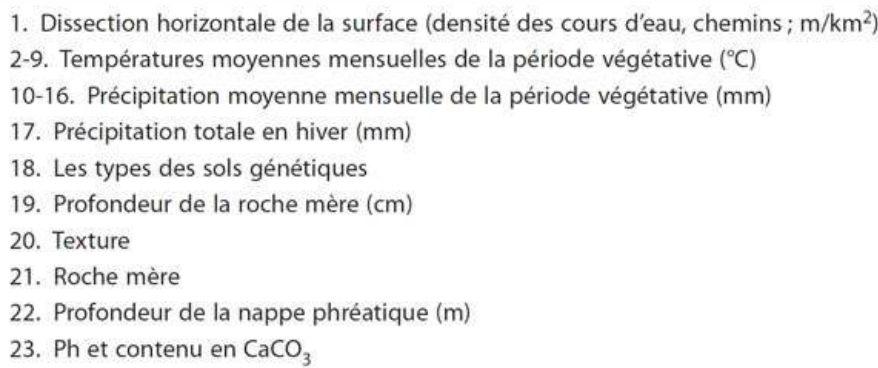

Ce type de carte donne une représentation très contrastée de la capacité productive du sol. L'échelle de l'utilité écologique des sols est évaluée en « degrés de convenance » de 0 (territoires construits ou qui ne conviennent pas) à 9 (territoires les mieux appropriés à la culture). Sur cette carte, pour pallier l'inconvénient cité plus haut (données antérieures à 1970), ce sont les facteurs indépendants du changement climatique, mais qui peuvent modifier ses conséquences, qui ont été pris en considération, comme les caractéristiques physiques des sols et leur composition granulométrique.

\section{La transformation de la couverture végétale et son rôle sur la sécheresse}

\section{Modification du couvert végétal}

La flore est naturellement influencée par les changements climatiques, et en premier lieu par la dégradation de l'approvisionnement en eau. On a longuement discuté pour savoir si l'apparition d'espèces végétales et animales thermophiles peut être considérée comme une preuve indirecte du changement climatique: plusieurs espèces de plantes thermophiles ont connu une expansion en Hongrie, mais il reste encore à en dresser un inventaire détaillé et à en connaître la signification écologique exacte, la notion de plante indicatrice du climat étant délicate à établir.

Sur le territoire modèle choisi entre Danube et Tisza, à côté des espèces continentales dominantes, le pourcentage de plantes qualifiées de "subméditerranéennes " à l'aide de divers indices (Borhidi, 1995) est de 35 à 40 \% (Kertész et al., 1998), et il est probable que ces espèces vont se propager sur un espace encore plus grand dans les prochaines années. Des enregistrements détaillés, couplés à des mesures de micro-climat, montrent que l'association Junipero-Populetum ouverte, vivant sur les sommets ou sur les versants des tertres sableux, descend progressivement vers les bas-fonds où l'humidité du sol reste accessible.

Un autre exemple est fourni par la restriction de l'aire de Quercus petrea, particulièrement dans les Massifs du Nord de la Hongrie. Cette disparition est attribuée à un manque d'azote absorbable dans le sol, consécutif à la sécheresse. A l'Ouest de la Transdanubie cependant, la restriction de l'aire de Quercus petrea est beaucoup moins marquée, et ceci peut être mis en relation avec le fait que la diminution des précipitations y est beaucoup moins grave que dans les Massifs du Nord. 


\section{Influence des forêts sur le régime des eaux} l'assèchement, combiné aux effets de l'activité humaine, se manifeste par l'abaissement 
du niveau des nappes phréatiques, l'épuisement des ressources en eaux profondes et par les changements induits dans l'utilisation du sol. Par rapport aux années 1960, le niveau annuel moyen des nappes phréatiques a baissé de 2 à 4 mètres.

Les conséquences du changement climatique doivent être prises en compte lors $d u$ choix des cultures, et il est donc nécessaire de déterminer la capacité productive de la région dans un avenir proche. L'évaluation du potentiel agro-écologique des différents espaces, déterminé par GIS, prend mieux en compte la réalité en incorporant les données des années 1980-2000, qui marquent une aggravation du processus (précipitations en baisse de 5 à $10 \mathrm{~mm} / \mathrm{an}$ ).

Si l'on se place à l'échelle de temps géochronologique, une période chaude et sèche pourrait être suivie d'une phase plus humide et plus fraîche. Mais dans le cadre plus rapide du changement climatique mondial actuel, il est convenable de supposer que l'activité humaine a intensifié la tendance au réchauffement, et ce de telle manière qu'elle a provoqué une transformation irréversible au sein du système climatique terrestre. Dans cette hypothèse, les caractères méditerranéens du climat de la Hongrie vont se renforcer et gagner de nouveaux territoires. Il faut donc dès maintenant envisager de nouvelles études dans le but d'observer les modifications probables des processus de pédogenèse, d'érosion et d'adaptation nouvelle de la couverture végétale et des cultures.

\section{BIBLIOGRAPHIE}

Borhidi A. (1995). - Social behaviour types, the naturalness and relative ecological indicator values of the higher plants in the Hungarian Flora. Acta. Bot. Hung., 39. 1-2, pp. 97-181.

Kertész Á., Papp S., Sántha A., Huszár T. and Lóczy D. (1998). - Impacts of aridification on soils and vegetation in a sand region of Hungary. Geografia fisica e dinamica quaternaria, Supplemento II (sous presse).

McRae S.G., Burnham C.P. (1981). - Land Evaluation. Clarendon Press, Oxford, 239 p. (Monographs on Soil Survey).

Molnár K. (1995). - Consequences of increasing aridity in the temperate zone - example : Hungary. Proceedings of International MEDALUS Meeting, 1995. Canary Islands.

Molnár K., Mika J. (1997). - Climate as a changing component of landscape : recent evidence and projections for Hungary. In : Bremer H., Lóczy D. (eds) : Geomorphology and changing environments in Central Europe. Zeitschrift für Geomorphologie, Supplementband 110, p. 185-195.

Várallyay Gy. (1994). - Climate change, soil salinity and alkalinity. In : Rounsevell, M.D.A. and Loveland, P.J. (eds.). Soil Response to Climate Change, NATO ASISeries, Springer-Verlag, Heidelberg, Ser. I. Vol. 23, p. 39-54. 
Varga-Haszonits Z., Harnos Zs. (1988). - Effect of climate variability and drought on wheat and maize production. In : Identifying and coping with extreme meteorological events. OMSZ,

Budapest, pp. 138-166.

\section{RÉSUMÉS}

Dans le cadre du programme MEDALUS de l'Union européenne, des recherches ont été effectuées sur l'aridification et ses conséquences dans le Bassin Carpathique. Les données climatiques observées sur les derniers 110 ans montrent en effet une tendance à la hausse des températures et à la baisse des précipitations, celle-ci étant le fait majeur, surtout depuis 1980. Les conséquences sont graves dans les régions collinaires (érosion des sols) et surtout sur les interfluves sableux (baisse importante des nappes souterraines, salinisation des sols). Ces données se répercutent sur la végétation et imposent la définition de nouvelles pratiques culturales.

Research has been carried out into aridification and its consequences in the Carpathian Basin, within the MEDALUS programme of the European Union. Climatic data from the last 110 years shows a trend towards rising temperatures and falling precipitation, the latter being the most important, especially since 1980 . The consequences are serious in hilly regions (soil erosion) and especially on sandy interluves (considerable lowering of water tables, salinisation of soils). These changes induce implications for vegetation and require new cultural practices.

Im Rahmen des MEDALUS-Programms der Europäischen Union wurden Untersuchungen über die Aridifizierung und ihre Folgen im Karpatenbecken durchgeführt. Die klimatischen Gegebenheiten der letzten 110 Jahre zeigen tatsächlich eine Tendenz zur Temperatur-zunahme und zur Nieder-schlagsabnahme, vornehmlich zu letzterer seit 1980. Die Folgen sind erheblich in den Hügelregionen (Bodenerosion) und vor allem in den sandigen Regionen zwischen den Flüssen (starke Grund-wasserabsenkung, Bodenversalzung). Diese Gegebenheiten wirken sich auf die Vegetation aus und erfordern die Bestimmung neuer Anbaupraktiken.

\section{INDEX}

Keywords : aridity, climatic change, ground water, Hungary, land-use, soil moisture

Mots-clés : agriculture, aridité, changement climatique, Hongrie, humidité des sols, nappe phréatique

Schlüsselwörter : Agrikultur, Aridität, Bodenfeuchtigkeit, Grundwasserspiegel, Klimawandel, Ungarn

\section{AUTEURS}

\section{ÁDÁM KERTÉSZ}

Académie des Sciences de Hongrie, Institut de Géographie Andrássy út 62,1062 Budapest, Hongrie

\section{DÉNES LÓCZY}

Académie des Sciences de Hongrie, Institut de Géographie Andrássy út 62,1062 Budapest, Hongrie 


\section{TAMÁS HUSZÁR}

Académie des Sciences de Hongrie, Institut de Géographie Andrássy út 62,1062 Budapest, Hongrie 